\title{
Tentativas de suicídio e suicídio em município de cultura Pomerana no interior do estado do Espírito Santo
}

\author{
Suicide attempts and suicide in a municipality of Pomerana culture \\ inside the state of Espirito Santo
}

Luciene Bolzam Macente', Elem Guimarães dos Santos², Eliana Zandonade

\section{RESUMO}

Objetivo: Descrever o perfil das tentativas de suicídio e mortalidade por suicídio no município de Santa Maria de Jetibá, interior do Espírito Santo, no período de 2001 a 2007. Método: Estudo descritivo, observacional e de abordagem quantitativa. A identificação dos casos de tentativas de suicídio e suicídios ocorreu por meio dos Boletins de Ocorrência Policial da Polícia Militar do Município, onde foram selecionados todos os casos. Para análise dos dados, foram utilizadas tabelas de frequência e os testes de hipótese $t$ Student e Qui-quadrado. Resultados: O coeficiente médio de mortalidade por suicídio foi de 12,9/100.000. Verificou-se que as mulheres realizam mais tentativas de suicídio, enquanto os homens foram mais eficientes, cometendo mais suicídio $(p=0,008)$. A média de idade para as vítimas de tentativas de suicídio foi de $30 \pm 10,1$ anos e de suicídio $42 \pm 14,9$ anos ( $p=0,001$ ). Os meios mais utilizados para os casos de suicídio foi o enforcamento (57,1\%), enquanto para as tentativas foi o envenenamento por agrotóxico (42,5\%). Conclusão: Verificou-se que o

\section{Palavras-chave}

Tentativas de suicídio, suicídio. perfil dos suicidas se diferencia dos que tentam, especialmente segundo o sexo, a idade e os meios utilizados, enfatizando a necessidade de se desencadear discussões acerca de programas, tratamento e prevenção para os grupos mais vulneráveis.

\section{ABSTRACT}

Objective: To describe the profile of suicide attempts and suicide mortality in the municipality of Santa Maria de Jetibá, inside Espírito Santo, from 2001 to 2007. Method: A descriptive, observational and quantitative study. The identification of cases of suicide attempts and suicides occurred through the police records from the Military Police of the city, where we selected all cases. Frequency tables were used and tests of hypotheses Student t and Chi-square in the data analysis. Results: The average mortality rate from suicide was 12.9/100,000. It was found that women carry out more suicide attempts, while men were more efficient, more committing suicide $(p=0.008)$. The average age for victims of suicide attempts was $30 \pm 10.1$ years and suicide $42 \pm 14.9$ years

1 Universidade Federal do Espírito Santo (UFES), Programa de Pós-graduação em Saúde Coletiva.

2 Universidade Federal do Espírito Santo (UFES), Programa de Pós-graduação em Saúde Coletiva; Núcleo de Estudos sobre Álcool e Drogas (NEAD/CCS/UFES). 


\section{Keywords}

Attempted suicide, suicide.
( $p=0.001)$. The most frequently used methods for cases of suicide was hanging (57.1\%), while for attempts was pesticide poisoning (42.5\%). Conclusion: It was found that the profile of suicidal person differs from the one who try, especially by gender, age and media used, emphasizing the need to initiate discussions about programs, treatment and prevention for vulnerable groups.

\section{INTRODUÇÃO}

Para a Organização Mundial de Saúde (OMS), suicídio constitui-se em "... um ato deliberado, iniciado e levado a cabo por uma pessoa com pleno conhecimento ou expectativa de um resultado fatal" ${ }^{\prime \prime}$. Verifica-se assim que se convencionou chamar de suicídio apenas as mortes em que a pessoa, voluntária e conscientemente, executou um ato ou adotou um comportamento, que acreditava levá-la à morte².

Segundo Werlang e Botega ${ }^{3}$, a palavra suicídio possui várias definições, cuja ideia central encontra-se no ato de "terminar com a própria vida", agregada a ideias menos evidentes relacionadas à motivação, à intencionalidade e à letalidade do ato. Poder-se-ia, contudo, considerar comportamento autodestrutivo (comportamento suicida) aqueles presentes em diversas condições, como uso de substâncias psicoativas, recusa a tratamento médico, determinados estilos de vida etc., os quais também podem resultar na morte, mas são mascaradas como se tivessem sido naturais.

Também as tentativas de suicídio fazem parte do que genericamente vem se chamando comportamento suicida, podendo estas serem definidas como atos intencionais de autoagressão que não resultam em morte. As tentativas de suicídio também são chamadas de autoagressão ou parassuicídio, englobando este termo atitudes e comportamentos variados, que envolvem desde atos mais graves, para os quais são necessárias hospitalizações, mas sem resultar em morte, até autoagressões que não necessitam de atendimento médico, o que dificulta a realização de estudos que abordem tal questão ${ }^{4}$.

Para a OMS, diversas circunstâncias podem aumentar o risco de suicídio por serem produtoras de estresse, como desemprego, pobreza, perda de uma pessoa querida, desentendimentos com familiares ou amigos, término de uma relação afetiva, problemas legais ou de trabalho. Sendo esses problemas corriqueiros em nossa vida, destaca que são poucas as pessoas que, ao enfrentá-los, cometem suicídio, por isso aponta a possibilidade de outros fatores influenciarem no processo de determinação do suicídio, tais como uso abusivo de álcool e drogas, disponibilidade dos meios para efetuar o ato suicida, violência física e/ou sexual na infância, isolamento social e distúrbios psíquicos, como a depressão, a esquizofrenia ou a falta de esperança $a^{5,6}$. Destaca-se, ainda, que o suicídio tem sido considerado uma prioridade pública em países de economia central, apresentando-se como uma das dez principais causas de morte entre todas as idades e entre as três causas na faixa etária de 15 e 35 anos $^{6}$.

Verifica-se assim que a mortalidade por suicídio caracteriza-se como um tema que desafia pesquisadores de diferentes áreas do conhecimento, por isso carece de maiores estudos, pois, quanto mais se puder entendê-la, mais fortes, eficazes e eficientes serão as ações já desenvolvidas, além de possibilitar o estabelecimento de novas políticas referentes a esse grave problema.

O objetivo desta pesquisa foi descrever o perfil das tentativas de suicídio e mortalidade por suicídio no município de Santa Maria de Jetibá, interior do Espírito Santo, no período de 2001 a 2007. O Município é constituído principalmente por descendentes de pomeranos, onde ainda hoje a cultura desse povo tem forte influência na região, podendo ser observada no meio de produção agrícola familiar, na religião luterana, na língua pomerana e em rituais como o casamento?. Esse município foi escolhido em decorrência dessas características, pois, com base em dados da literatura, os fatores geográficos e culturais, relacionados às estatísticas de suicídio, são importantes e devem ser investigados, pois possibilitam desenvolver explicações multifacetadas, por meio de macroanálises, sobre as principais evidências das causas de tal fenômeno ${ }^{8}$.

\section{METODOLOGIA}

Estudo descritivo, observacional e de abordagem quantitativa, visando descrever o perfil da mortalidade por suicídio e das tentativas de suicídio no município de Santa Maria de Jetibá no período de 2001 a 2007. Esse município é um dos que compõem a região serrana do Espírito Santo, está localizado a 80 quilômetros da capital Vitória e possui uma população de aproximadamente 33.468 habitantes ${ }^{9}$, a qual se encontra majoritariamente em meio rural.

Os dados referentes às tentativas de suicídio e suicídios para o período foram obtidos por meio da identificação das vítimas pelos Boletins de Ocorrência Policial (BOP) da Polícia Militar do Município, sendo selecionados 100\% dos casos identificados. Foram utilizados os códigos 104 para tentativa e 103 para suicídio.

As variáveis de estudo foram selecionadas conforme disposição das informações referentes às vítimas nos BOP's, tanto para os casos de tentativa de suicídios, quanto para os ca- 
sos de suicídios: dia da semana; turno das ocorrências; sexo; idade; profissão; meios utilizados e os motivos para o ato.

Para análise dos dados foi utilizado o teste $t$ Student e o teste Qui-quadrado. Quanto à apresentação dos resultados, estes ocorreram por meio de tabelas e gráficos, visando à melhor compreensão destes. O nível de significância adotado foi de $5 \%$.

No que se refere aos aspectos éticos, destaca-se que todo o processo de pesquisa foi conduzido conforme a Resolução No. 196/96 do Conselho Nacional de Saúde (CNS), tendo sido o projeto aprovado pelo Comitê de Ética e Pesquisa da Universidade Federal do Espírito Santo.

\section{RESULTADOS}

No período analisado (2001 a 2007) foram encontrados 80 BOP's referentes a casos de tentativas de suicídio e 28 referentes a casos de suicídios no município de Santa Maria de Jetibá, o que corresponde a um coeficiente de mortalidade médio de 12,9/100.000 e média de 11,4 tentativas de suicídio/ano. Destaca-se que não foram observadas mais de uma tentativa de suicídio por indivíduo.

No que se refere ao coeficiente de mortalidade, conforme figura 1, verificou-se oscilações no período analisado que vão desde a maior taxa em 2002, com 23,38/100.000, até a menor taxa em 2005, com 3,1/100.000.

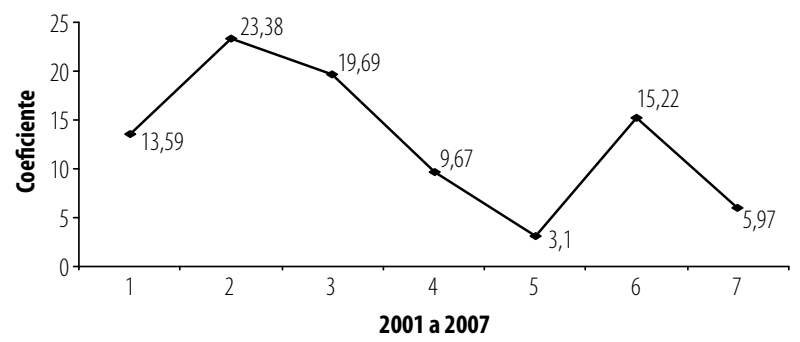

Figura 1. Distribuição do coeficiente de mortalidade no período analisado.

Os dias da semana com maior número de ocorrências para as tentativas de suicídio foram o domingo $(30,0 \%)$ e a segunda-feira (16,25\%), enquanto, para os suicídios, observase que a segunda $(21,42 \%)$, a quarta e a sexta-feira $(17,85 \%$ para ambos) foram os dias de maior ocorrência.

Ao se comparar tentativa e suicídio segundo sexo, verificou-se significância estatística ( $p=0,008)$, conforme tabela 1 , para o fato de as mulheres terem cometido mais tentativas de suicídio que os homens, contudo, destaca-se que estes últimos foram mais eficientes e com isso cometeram mais suicídios que elas.
Tabela 1. Distribuição dos casos de tentativas de suicídio e de suicídios segundo sexo

\begin{tabular}{cccccccc}
\hline & \multicolumn{9}{c}{ Evento } \\
\cline { 2 - 8 } & & \multicolumn{2}{c}{ Suicídio } & \multicolumn{2}{c}{ Tentativa } & \multicolumn{2}{c}{ Total } \\
\cline { 2 - 8 } & No & $\%$ & No & $\%$ & $N^{\circ}$ & $\%$ \\
\hline \multirow{2}{*}{ SEX0 } & F & 3 & 10,7 & 30 & 37,5 & 33 & 30,6 \\
& M & 25 & 89,3 & 50 & 62,5 & 75 & 69,4 \\
\hline
\end{tabular}

Qui-quadrado $=7,013 ; p=0,008$.

Quanto à média de idade, houve diferença entre as vítimas de tentativas de suicídio (30 $\pm 10,1$ DP anos) e de suicídios (42 $\pm 14,9$ DP anos) ( $p=0,001$ ), conforme a figura 2 . Quando a idade foi estratificada, evidenciou-se que 41,3\% das vítimas que tentaram suicídio encontravam-se entre os 25 e 35 anos, enquanto, entre as vítimas de suicídio, 32,1\% encontravam-se na faixa etária de 35 a 50 anos e outros $32,1 \%$, acima de 50 anos. Esse resultado aponta uma menor faixa etária entre as vítimas de tentativas de suicídio do que entre as vítimas de suicídio $(p=0,002)$.

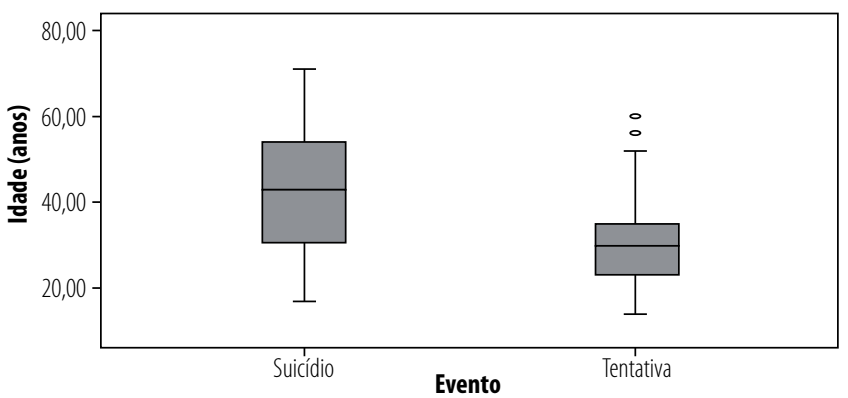

Figura 2. Distribuição dos casos de tentativas de suicídio e de suicídios, de acordo com a faixa etária Box plot (quartis, mínimo e máximo) das idades em anos segundo evento suicídio e tentativa.

Comparando as variáveis faixa etária e sexo em ambos os eventos separadamente, não foi identificada diferença para os casos de suicídio, contudo evidenciou-se diferença para as tentativas de suicídio ( $p=0,001)$, conforme observado na tabela 2. Nota-se que, entre as vítimas de tentativas de suicídio, as mulheres possuem idade inferior a dos homens.

Tabela 2. Comparação das variáveis faixa etária e sexo para o evento tentativas de suicídio

\begin{tabular}{|c|c|c|c|c|c|c|}
\hline \multirow{3}{*}{ Faixa etária } & \multicolumn{4}{|c|}{ Sexo } & \multirow{2}{*}{\multicolumn{2}{|c|}{ Total }} \\
\hline & \multicolumn{2}{|c|}{$\mathbf{F}$} & \multicolumn{2}{|c|}{ M } & & \\
\hline & No & $\%$ & No & $\%$ & No & $\%$ \\
\hline$<18$ & 7 & 23,3 & 1 & 2,0 & 8 & 10,0 \\
\hline $18 \mid---25$ & 8 & 26,7 & 7 & 14,0 & 15 & 18,8 \\
\hline $25 \mid--35$ & 12 & 40,0 & 21 & 42,0 & 33 & 41,3 \\
\hline $35 \mid---50$ & 3 & 10,0 & 17 & 34,0 & 20 & 25,0 \\
\hline Acima dos 50 anos & 0 & 0 & 4 & 8,0 & 4 & 5,0 \\
\hline Total & 30 & 100,0 & 50 & 100,0 & 80 & 100,0 \\
\hline
\end{tabular}

Qui-quadrado $=18,924 ; p=0,001$. 
Tabela 3. Métodos utilizados pelas vítimas de tentativas de suicídio e de suicídio

\begin{tabular}{|c|c|c|c|c|c|c|}
\hline \multirow{3}{*}{ Meios utilizados } & \multicolumn{6}{|c|}{ Evento } \\
\hline & \multicolumn{2}{|c|}{ Suicídio } & \multicolumn{2}{|c|}{ Tentativa } & \multicolumn{2}{|c|}{ Total } \\
\hline & $N^{0}$ & $\%$ & No & $\%$ & $\mathrm{~N}^{0}$ & $\%$ \\
\hline Afogamento & 0 & .0 & 2 & 2,5 & 2 & 1,9 \\
\hline Arma de fogo & 2 & 7,1 & 4 & 5,0 & 6 & 5,6 \\
\hline Enforcamento & 16 & 57,1 & 3 & 3,8 & 19 & 17,6 \\
\hline Envenenamento - medicamentos & 0 & 0 & 8 & 10,0 & 8 & 7,4 \\
\hline Envenenamento & 6 & 21,4 & 22 & 27,5 & 28 & 25,9 \\
\hline Envenenamento agrotóxico & 2 & 7,1 & 34 & 42,5 & 36 & 33,3 \\
\hline Faca & 1 & 3,6 & 3 & 3,8 & 4 & 3,7 \\
\hline Jogar-se de altura & 0 & 0 & 3 & 3,8 & 3 & 2,8 \\
\hline Sem informação & 1 & 3,6 & 1 & 1,3 & 2 & 1,9 \\
\hline Total & 28 & 100,0 & 80 & 100,0 & 108 & 100,0 \\
\hline
\end{tabular}

Qui-quadrado $=47,01 ; p=0,001$.

No que se refere aos meios utilizados nos eventos, observa-se diferença estatística $(p=0,001)$, conforme a tabela 3 . Enquanto para os casos de suicídio o mais usual foi o enforcamento $(57,1 \%)$, para as tentativas de suicídio destacou-se o envenenamento por agrotóxico (42,5\%).

\section{DISCUSSÃO}

Observa-se que os óbitos associados com suicídio fazem parte do banco de dados sobre mortalidade da OMS, o que significa dizer que, em todos os conjuntos de notificações de todos os países sobre mortalidade, existe informação sobre suicídio, mas contrariamente a isso, no que se refere às informações referentes às tentativas de suicídio, a OMS não obriga tal notificação dos casos, por isso em praticamente nenhum país se coleta tal dado de forma sistemática ${ }^{10}$.

Um exemplo é o estudo de Souza et al. ${ }^{11}$ desenvolvido em Itabira, onde se identificou, com os dados do Sistema de Informações Hospitalares do Sistema Único de Saúde (SIH-SUS), a ocorrência de apenas duas internações por tentativas de suicídio naquele Município no ano de 2000 (em ambos os casos as vítimas eram homens na faixa dos 15 aos 24 anos de idade e o traumatismo foi a lesão que gerou a necessidade de internação nos dois casos), o que representavam 2,0\% do total das 100 internações por causas externas nesse ano. Contudo, ao buscarem os dados do Batalhão da Polícia Militar, descobriram a existência de 539 tentativas de suicídio no período de 1996 a 2001, dos quais 124 ocorreram em 2000. Essa discrepância pode sugerir que apenas os casos mais graves acabam levando à internação hospitalar e, com isso, boa parte dos que tentaram suicídio acaba atendida nas emergências e, assim, o caso não é registrado nas internações, as quais geram a Autorização de Internação Hospitalar (AlH).

Calcula-se que mais de dois terços dos suicídios ocorrem na primeira tentativa, contudo, a maioria das tentativas possui baixa letalidade, sendo motivada, geralmente, pela ocorrência de uma crise interpessoal ou social ${ }^{12}$. Estima-se que as taxas de tentativas de suicídio são de 5 a 20 vezes maiores que as de suicídio, ocorrendo mais frequentemente em jovens e mulheres, apesar da observação de que essa diferença de gênero venha diminuindo ${ }^{12-14}$.

Em relação ao coeficiente de mortalidade, a presente pesquisa mostrou uma variação que foi desde a maior taxa em 2002, com 23,38/100.000, até a menor taxa em 2005, com 3,1/100.000. Segundo Mello ${ }^{4}$, esse coeficiente é considerado baixo quando menor que 5/100.000, médio entre 5 e 15/100.000, alto entre 15 e 30/100.000 e muito alto quando superior a 30/100.000, o que nos leva a não poder identificar um padrão para o Município. Contudo, chama a atenção os dados do Relatório da OMS ${ }^{15}$, segundo o qual a média para 53 países onde há dados completos disponíveis, a taxa agregada e padronizada de suicídio para o ano de 1996 foi de 15,1 por 100.000, mostrando assim que o Município chegou a possuir taxas bem maiores.

Quanto ao dia da semana, Kliemann ${ }^{16}$, ao desenvolver um estudo com o objetivo de descrever o perfil epidemiológico dos óbitos por suicídio ocorridos na região da grande Florianópolis, no período de 1991 a 2005, encontrou resultado diferente dessa pesquisa, ao identificar que uma maior proporção de suicídios ocorreu na quinta-feira com 112 óbitos (16,7\%), seguido pelo domingo com 106 óbitos (15,8\%), enquanto neste estudo foi encontrado que o maior número de casos ocorreu na segunda $(21,42 \%)$ e na quarta e sextafeira (17,85\% para ambos). 
Já quanto ao sexo, observa-se que os resultados encontrados estão em conformidade com os dados disponíveis na literatura, em que se observa o predomínio das tentativas de suicídio entre o sexo feminino, em contraste com o ato suicida, o qual é mais frequente no sexo masculino ${ }^{11,17,18}$.

A menor ocorrência de suicídio entre as mulheres tem sido atribuída a vários fatores, como a baixa prevalência de dependência do álcool, a maior religiosidade, o envolvimento social e o desempenho de papéis durante a vida. Além disso, as mulheres conseguem reconhecer mais precocemente sinais de riscos para depressão, suicídio e doença mental, além de buscarem mais ajuda em momentos de crise e de participarem mais das redes de apoio social. Quanto aos homens, estes desempenham comportamentos que predispõem ao suicídio (a competitividade, a impulsividade e o maior acesso a tecnologias letais), sendo ainda mais sensíveis às instabilidades econômicas, como desemprego e empobrecimento etc., fatores esses que podem levar ao suicídio ${ }^{19,20}$.

Também Hesketh e Castro ${ }^{21}$ destacam a tese de que o suicídio é um ato de caráter mais final para o homem do que para a mulher, pois, enquanto o homem busca no ato extremo do suicídio a solução drástica para seu fracasso pessoal, principalmente sob o prisma socioeconômico, a mulher tenta por meio do suicídio remediar condições adversas de natureza essencialmente afetiva.

Em relação à faixa etária entre as vítimas de suicídio, pesquisa realizada por Baptista e Borges ${ }^{22}$, com o objetivo de avaliar algumas características demográficas e sociais referentes aos suicidas por meio de laudos do IML de Limeira, entre os anos de 1998 e 2002, encontrou-se uma média de idade de 39,3 anos para as vítimas de suicídio. No estudo de Kliemann ${ }^{16}$, essa média foi de 39,4 anos entre as vítimas de suicídio, enquanto Ponce et al. ${ }^{18}$, em estudo realizado com o objetivo de determinar a prevalência de alcoolemia positiva entre as vítimas de suicídios necropsiadas nos postos médico-legais do Estado de São Paulo, durante o ano de 2005, encontraram uma média de idade entre todas as vítimas de suicídio de 39,8 anos. Observa-se, assim, uma média de idade das vítimas de suicídio menor do que a encontrada na presente pesquisa.

Quanto à faixa etária das vítimas de tentativas de suicídio, observa-se que os resultados da presente pesquisa encontram-se em conformidade com os apontados por vários pesquisadores: Hesketh e Castro ${ }^{21}$ encontraram maior frequência de tentativas de suicídio entre jovens de até 25 anos. Souza et al. ${ }^{11}$ identificaram faixas etárias jovens de 15 a 24 anos (29,7\%) e de 25 a 39 anos (34,4\%); Miranda e Queiroz ${ }^{23}$ destacam que $90 \%$ das tentativas de suicídio em Belo Horizonte se dão entre 15 e 44 anos. Cassorla ${ }^{24}$, em um estudo clínico-epidemiológico, tipo caso-controle, em que o grupo de casos foi constituído por 50 jovens que haviam tentado suicídio, identificou que a análise e a projeção do número de casos de tentativas de suicídio atendidos em pronto-socorro foram de 75\% em menores de 27 anos; Schmitz et al. ${ }^{19}$, em sua pesquisa, destacam como as faixas etárias mais prevalentes as de 25 a 39 anos e a de 17 a 24 anos, perfazendo as duas somadas $68,8 \%$ de todas as tentativas de suicídio por envenenamento.

Segundo Meneghel et al. ${ }^{20}$, os coeficientes de mortalidade por suicídio aumentam com a idade, afirmação essa que coincide com os resultados encontrados na presente pesquisa. Contudo, eles também afirmam que o padrão etário está mudando, apontando para uma sociedade em que há poucas perspectivas para a população mais jovem. No Rio Grande do Sul, onde se observam os maiores coeficientes de mortalidade por suicídio, o grupo etário de maior ocorrência é entre a população idosa (maior de 70 anos), porém nos últimos anos da década de 1990 apareceu uma tendência de elevação nas faixas mais jovens, dos 20 aos 59 anos, o que pode ser observado no resultado de várias outras pesquisas: Kliemann ${ }^{16}$, ao considerar o período de 1991 a 2005 a distribuição das faixas etárias entre as vítimas de suicídio, mostrou a maior proporção para o intervalo dos 20 aos 29 anos com 24,7\% óbitos, seguida pela faixa dos 30 aos 39 anos, com $22,8 \%$ casos; Baptista e Borges ${ }^{22}$ encontraram que a maior prevalência de suicídios ocorreu na faixa etária dos 21 aos 40 anos, seguida pela meia-idade, adolescência e terceira idade, em ambos os sexos; Parente et al. ${ }^{17}$, ao analisarem a faixa etária, observaram a presença de mortes por suicídio em todas essas, sendo a incidência maior nas populações mais jovens; Miranda e Queiroz ${ }^{23}$, em seu estudo em Belo Horizonte, encontraram maiores coeficientes de mortalidade por suicídio nas faixas etárias dos 15 aos 24 anos e dos 25 aos 44 anos, observando que no período estudado houve um crescimento do coeficiente na faixa etária dos 15 aos 44 anos.

Pensando na interação entre sexo e idade, os resultados encontrados estão em consonância com as afirmações de Hesketh e Castro21, segundo as quais as mulheres mais jovens e os homens com mais idade apresentam maior número de tentativas.

No que se refere aos meios utilizados, Lopes ${ }^{25}$, ao analisar as diferenciações sexuais constantes e recorrentes em discursos médicos do século XIX, entre o suicídio cometido por homens e mulheres, destaca que pelo que parece as escolhas e os meios utilizados para se dar à morte é um importante fator para se compreender a constituição do suicídio entre homens e mulheres, pois traria em si os signos da masculinidade e da feminilidade. Nesse sentido, os homens prefeririam se suicidar utilizando a estrangulação, a arma de fogo e as chamadas armas brancas, enquanto as mulheres prefeririam a asfixia por carbono, o envenenamento e a queda de um lugar mais elevado, visto buscarem uma morte mais "lenta e doce".

São várias as pesquisas que comprovam tais colocações, tanto para os casos de suicídio quanto para as tentativas. 
Baptista e Borges $^{22}$ encontraram que, entre as vítimas de suicídio, os meios mais utilizados pelos homens foram o enforcamento ( $56 \%$ dos casos); a arma de fogo (24\%) e a intoxicação por veneno (7,2\%), enquanto os meios mais utilizados pelas mulheres foram o enforcamento (39,3\%), seguido de intoxicação/veneno $(21,4 \%)$ e queimaduras $(17,9 \%)$. Parente et al. ${ }^{17}$ destacam que os meios mais utilizados nos cinco anos pesquisados foi o enforcamento (66\%), seguido de arma de fogo $(13,1 \%)$ e envenenamento $(11,9 \%)$. Ponce et al. ${ }^{18}$ encontraram que o principal agente causador da morte entre as mulheres foi a intoxicação (28,2\%), sendo 56,9\% por carbonatos, seguido do enforcamento (23,3\%). Entre os homens, o principal agente foi o enforcamento (34,3\%) e o segundo foi ferimento por arma de fogo (14,9\%), o que se pode verificar assim que, em geral, as mulheres optam por envenenamento ou ingestão de medicamentos, enquanto os homens buscam métodos mais violentos e letais, como enforcamento e arma de fogo.

Para as tentativas de suicídio, podem-se citar os dados obtidos por Hesketh e Castro21, os quais encontraram maior incidência de problemática afetiva nas mulheres suicidas; maior incidência de problemática socioeconômica entre os homens e maior intencionalidade nas tentativas de suicídio entre estes últimos. Miranda e Queiroz ${ }^{23}$ encontraram, entre os meios utilizados nas tentativas de suicídio, que o método preferido é o de envenenamento por substâncias tóxicas sólidas ou líquidas; o segundo meio empregado são os classificados como outros e a terceira escolha é a arma branca. Schmitz et al..$^{19}$ identificaram que, das substâncias usadas nas tentativas de suicídio, os agrotóxicos representaram 17,6\%; se somados aos pesticidas veterinários (ambos facilmente encontrados no meio rural, como é o caso da presente pesquisa), chega a 19,7\% a taxa de uso dessas substâncias. Os psicofármacos representaram 31,7\%; considerando-se todos os medicamentos, a taxa chegou a 47,5\%.

Apesar das limitações desta pesquisa, entre elas a inexistência de dados oficiais referentes às tentativas de suicídio, os resultados demonstram a importância de estudos em realidades diferenciadas, como a do município de Santa Maria de Jetibá, pois, conforme afirma Meneghel et al. ${ }^{20}$, quando o suicídio acontece preponderantemente em um grupo etário, étnico, profissional ou isolado geograficamente, como é o caso deste Município, deve-se indagar se esse evento estaria funcionando como um indicador de pressão na sociedade.

\section{CONCLUSÃO}

Os resultados encontrados mostram-se em consonância com dados da literatura sobre o tema, na medida em que apontou diferenças significativas entre as vítimas de tentativas de suicídios e de suicídio, principalmente em relação à faixa etária, ao sexo e ao meio utilizado, o que sugere que tais fenômenos agrupam indivíduos com características peculiares. Aponta-se, assim, a necessidade de se desencadear discussões acerca de programas, tratamento e prevenção para os grupos mais vulneráveis, os quais podem e devem ser detectados e acompanhados tanto por programas da atenção básica de saúde quanto por aqueles da rede especializada.

Evidenciou-se ainda a importância de se implantar um rigoroso controle sobre a prescrição e a distribuição de medicamentos (principalmente psicofármacos) e sobre o uso de agrotóxicos (com a utilização de agrotóxicos de menor toxicidade), pois essas são medidas fundamentais para uma diminuição das taxas de autoenvenenamento. Outra questão evidenciada é a necessidade de se melhorar a qualidade dos dados disponíveis ${ }^{26}$, pois os dados oficiais, especialmente os que se referem às tentativas de suicídio no Brasil, ainda carecem de um sistema confiável, sem o qual se pode distorcer a realidade desses fenômenos $s^{9,27,28}$.

Outra questão é a necessidade de se desenvolverem novos estudos sobre o tema, principalmente com foco em populações diferenciadas como a do presente estudo, pois apenas assim será possível subsidiar as intervenções/ações profissionais, de forma a aproximá-las o máximo de uma realidade local, a qual é multifacetária e complexa, possibilitando, assim, o desenvolvimento de medidas de prevenção mais eficazes.

\section{REFERÊNCIAS}

1. Organização Mundial de Saúde. Relatório sobre a saúde no mundo 2001: saúde mental nova concepção, nova esperança. Genebra. 2001:66

2. Cassorla RMS. Suicídio e autodestruição humana. In: Werlang BG, Botega NJ, organizadores. Comportamento suicida. Porto Alegre: Artmed; 2004. p. 21-33.

3. Werlang BSG, Botega NJ, organizadores. Comportamento suicida. 1. ed. Porto Alegre: Artmed; 2004

4. Mello MF. Epidemiologia da saúde mental no Brasil. 1. ed. Porto Alegre: Artmed; 2007.

5. De Leo D. In: Werlang BG, Botega NJ, organizadores. Comportamento suicida. Porto Alegre: Artmed; 2004. p. 13-16.

6. Werneck GL, et al. Tentativas de suicídio em um hospital geral no Rio de Janeiro, Brasil. Cad. Saúde Pública. 2006;22(10):2201-6.

7. Rölke HR. Descobrindo raízes, aspectos geográicos, históricos e culturais da Pomerânia. Vitória: UFES. Secretaria de Produção e Difusão Cultural; 1996.

8. Baptista MN. Suicídio e depressão - Atualizações. Rio de Janeiro: Editora Guanabara Koogan; 2004.

9. IBGE. Instituto Brasileiro de Geografia e Estatística. Contagem da População 2008 [base de dados na internet]. Disponível em: http://www.ibge.gov.br/home/estatistica/populacao/ estimativa2008/POP_2008_TCU.pdf. Acesso em: 8 de março de 2009.

10. Bertolote JM, Fleischmann A. Suicídio e doença mental: uma perspectiva global. In: Werlang BG, Botega NJ, organizadores. Comportamento suicida. Porto Alegre: Artmed; 2004. p. 35-44.

11. Souza ER, Minayo MCS, Cavalcante FG. 0 impacto do suicídio sobre a morbimortalidade da população de Itabira. Ciên Saúde Colet. 2007;11(Sup):1333-42.

12. Mann JJ. A current perspective of suicide and attempted suicide. Annals of Internal Medicine. 2002;136(4):302-11. 
13. Gunnell DJ. The epidemiology of suicide. Int Rev Psychiatry. 2000;12:21-6.

14. Bahls SC, Bahls FRC. Depressão na adolescência: características clínicas. Interação Psicologia. 2002;6(1):49-57.

15. OMS. Organização Mundial de Saúde. Relatório sobre a saúde no mundo 2001: saúde mental - Nova concepção, nova esperança. Genebra; 2001.

16. Kliemann DVO. Estudo epidemiológico de óbitos por suicídio na região da grande Florianópolis de 1991 a 2005 [trabalho de conclusão de curso]. Santa Catarina: Universidade Federal de Santa Catarina. Curso de Medicina, 2007.

17. Parente ACM, Soares RB, Araújo ARF, Cavalcante IS, Monteiro CFS. Caracterização dos casos de suicídio em uma capital do Nordeste Brasileiro. Rev Bras Enferm. 2007;60(4):377-81.

18. Ponce JC, Andreuccetti G, Jesus MGS, Leyton V, Muñoz DR. Álcool em vítimas de suicídio em São Paulo. Rev Psiquiatr Clín. [online]. 2008;35 (suppl.1):13-16.

19. Schmitz M, Torres JB, Soares PFB. Tentativas de suicídio por auto-envenenamento: um estudo sobre 684 casos. Revista ABP-APAL. 1992;14(2):63-6.

20. Meneghel SN, Victora CG, Faria NMX, Carvalho LA, Falk JW. Características epidemiológicas do suicídio no Rio Grande do Sul. Rev Saúde Pública [online]. 2004;38(6):804-10.
21. Hesketh JL, Castro AG. Fatores correlacionados com a tentativa de suicídio. Rev Saúde Pública. 1978;12:138-46.

22. Baptista MN, Borges A. Suicídio: aspectos epidemiológicos em Limeira e adjacências no período de 1998 a 2002. Estud Psicol. 2005;22(4):425-31.

23. Miranda PSC, Queiroz EA. Suicídio e tentativas de suicídio: características epidemiológicas no município de Belo Horizonte - Brasil, 1971-81. Arq Neuro-Psiquiat. 1989;47(4):403-12.

24. Cassorla RMS. Características das famílias de jovens que tentaram suicídio em Campinas, Brasil: um estudo comparativo com jovens normais e psiquiátricos. Acta Psicol Amér Lat. 1984;30:125-34

25. Lopes FH. Medicina, educação e gênero: as diferenciações sexuais do suicídio nos discursos médicos do século XIX. Educ Rev [online]. 2007;29:241-57.

26. Grossi R, Vansan GA. Mortalidade por suicídio no município de Maringá (PR). J Bras Psiquiatr. 2002;51(2):101-11.

27. Botega NJ, Rapeli CB. Tentativa de suicídio envolvendo risco de vida: internações em um hospital geral. J Bras Psiquiatr. 1998;44(1):157-62.

28. Silva JA, Silva CN, Silva Jr, Silva LN, Silva DN. Epidemiologia do suicídio na cidade de Salvador (BA). Rev Bras Neurol Psiquiátr. 1999;3(1):19-25. 